\title{
CAFFEINE AND ADENOSINE A $2 A$ RECEPTOR INACTIVATION DECREASE STRIATAL NEUROPATHOLOGY IN A LENTIVIRAL-BASED MODEL OF MACHADO-JOSEPH DISEASE
}

\footnotetext{
Running head: Caffeine alleviates MJD-striatal neuropathology
}

Nélio Gonçalves, MSc ${ }^{1,2}$, Ana T. Simões, MSc ${ }^{1,2}$, Rodrigo A. Cunha, PhD ${ }^{1,3}$, Luís Pereira de Almeida, $\mathrm{PhD}^{1,2}$

From the ${ }^{1} \mathrm{CNC}-$-Center for Neuroscience and Cell Biology, University of Coimbra, 3004-517 Coimbra, Portugal; ${ }^{2}$ FFUC - Faculty of Pharmacy, University of Coimbra, 3000-548 Coimbra, Portugal; ${ }^{3}$ FMUC - Faculty of Medicine, University of Coimbra, 3004-504 Coimbra, Portugal

Address correspondence to Luís Pereira de Almeida, CNC -Center for Neuroscience and Cell Biology, University of Coimbra, 3004-517 Coimbra, Portugal. Ph: +351 239820190, Fax: +351 239822776; e-mail: luispa@ci.uc.pt.

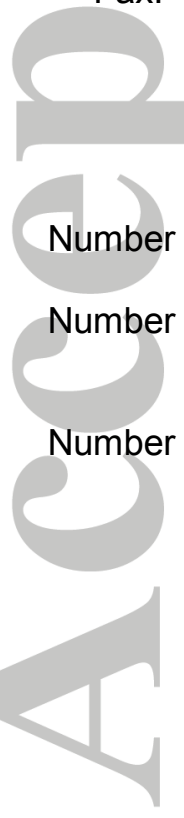

This article has been accepted for publication and undergone full peer review but has not been through the copyediting, typesetting, pagination and proofreading process which may lead to differences between this version and the Version of Record. Please cite this article as an 'Accepted Article', doi: 10.1002/ana.23866 


\section{Abstract}

Objective:

Machado-Joseph disease (MJD) is a neurodegenerative disorder associated with an abnormal CAG expansion, which translates into an expanded polyglutamine tract within ataxin-3. There is no therapy to prevent or modify disease progression. Since caffeine (a non-selective adenosine receptor antagonist) and selective adenosine $A_{2 A}$ receptor $\left(A_{2 A} R\right)$ blockade alleviate neurodegeneration in different brain diseases, namely at early stages of another polyglutamine-related disorders such as Huntington's disease, we now tested their ability to control MJD-associated neurodegeneration.

\section{Methods:}

MJD was modelled by transducing the striatum of male adult C57BI6 mice with lentiviral vectors encoding mutant ataxin- 3 in one hemisphere and wild-type ataxin- 3 in the other hemisphere (as internal control). Caffeine $(1 \mathrm{~g} / \mathrm{L})$ was applied through the drinking water. Mice were killed at different time points (from 2-12 weeks) to probe for the appearance of different morphological changes using immunohistochemical analysis.

\section{Results:}

Mutant ataxin-3 caused an evolving neuronal dysfunction (loss of DARPP-32 staining) leading to neurodegeneration (Cresyl violet and NeuN staining) associated with increased number of mutant ataxin-3 inclusions in the basal ganglia. Notably, mutant ataxin-3 triggered early synaptotoxicity (decreased synaptophysin and MAP-2 staining) and reactive gliosis (GFAP and CD11b staining), which predated neuronal dysfunction and damage. Caffeine reduced the appearance of all these morphological modifications, which were also abrogated in mice with a global $A_{2 A} R$ inactivation (knockout).

\section{Interpretation:}

Our findings provide a demonstration that synaptotoxicity and gliosis are precocious events in MJD and that caffeine and $A_{2 A} R$ inactivation decrease MJD-associated striatal pathology, which paves the way to consider $A_{2 A} R$ as novel therapeutic targets to manage MJD. 


\section{Introduction}

Various inherited neurodegenerative diseases result from an increase in the number of CAG codon repeats within the open reading frame of the responsible gene. ${ }^{1}$ MachadoJoseph disease (MJD) or spinocerebellar ataxia type 3 , is one such dominantly-inherited polyglutamine neurodegenerative disease and the most common among ataxias. ${ }^{2}$ It is characterised by an adult age of onset causing premature death associated with unstable expansion of a CAG stretch (over 55 repeats) in the MJD1 gene that encodes a polyglutamine repeat in the corresponding ataxin-3 protein., ${ }^{3,4}$ The clinical hallmarks of MJD include progressive ataxia, dysfunction of motor coordination, postural instability and Parkinsonism among other symptoms. ${ }^{5,6}$ The neuropathology of MJD involves multiple systems such as cerebellar systems, substantia nigra and cranial nerve motor nuclei, ${ }^{3,7}$ as well as the striatum..$^{8-11}$ Degeneration and loss of neuronal cells in MJD is accompanied by the presence of protein aggregates, ${ }^{12}$ designated as neuronal intranuclear inclusion bodies (NIIs). Although transgenic animal models closely mimicking the human pathology, ${ }^{8,13-15}$ have bolstered our understanding of MJD, the mechanisms accounting for neuronal degeneration are still largely unknown. Albeit not yet explored in MJD, studies in other polyQ disorders suggest that neuronal dysfunction and synaptotoxicity may precede degeneration and appearance of clinical symptoms, ${ }^{16,17}$ and that neuroinflammation, ${ }^{18}$ may function as an amplificatory loop exacerbating neuronal damage. ${ }^{19-21}$

There is currently no therapy to manage MJD. We posed that chronic caffeine consumption, which affords neuroprotection through the antagonism of adenosine $A_{2 A}$ receptors $\left(A_{2 A} R\right),{ }^{22}$ might be a candidate strategy to manage MJD neurodegeneration. Caffeine and $A_{2 A} R$ blockade afford robust neuroprotection in different neurodegenerative disorders, in accordance with the key role of $A_{2 A} R s$ controlling synaptic viability, apoptotic neuronal death, astrocytic function and neuroinflammation. ${ }^{23,24}$ In particular, in Huntington's disease, another poly $Q$ disorder, $A_{2 A} R$ blockade at the prodrome or early stages of the disease seems to delay the appearance of clinical symptoms, mainly through a normalization 
of striatal glutamatergic transmission, which impedes the characteristic degeneration of striatal neurons. ${ }^{25}$ Importantly, it has recently been shown in induced pluripotent stem cellsderived neurons that glutamate overstimulation raises intracellular calcium levels activating the cysteine proteases calpains and promoting the proteolysis and aggregation of ataxin- $3 .^{26}$ Moreover, calpain-mediated proteolysis of ataxin-3 in a rodent model of MJD mediates translocation of ataxin-3 to the cell nucleus, aggregation and neurodegeneration, which can be prevented by calpain inhibition. ${ }^{27}$

In view of this proposed key role of glutamate overstimulation in MJD, we now investigated the time course of neuropathological modifications in a genetic model of MJD and tested the novel hypothesis that the manipulation of $A_{2 A} R$ function might also be beneficial in MJD.

\section{Materials and Methods}

\section{Animals}

Male C57BL/6 mice (Charles River, Barcelona, Spain) were housed and kept under a conventional 12-h light-dark cycle maintained on a temperature-controlled room with food and water provided ad libitum and used at 7 weeks of age. C57BI/6-background $A_{2 A} R$ knockout $\left(A_{2 A} R K O\right)$ and age-matched wild type control mice were obtained from parallel breeding of our colony of $A_{2 A} R$ KO mice, initially obtained from J.F.Chen (Boston University). ${ }^{28}$ The experiments were carried out in accordance with the European Community directive (86/609/EEC) for the care and use of laboratory animals.

\section{Drug treatment}

We chose the dose of caffeine $(1 \mathrm{~g} / \mathrm{L})$ administered through the drinking water, as a maximally effective and non-toxic dose, which we have previously shown to result in a plasma concentration of $50 \mu \mathrm{M},{ }^{29}$ and similar concentration in the brain parenchyma, ${ }^{30}$ corresponding to a diary human consumption of circa 5 cups of coffee. Treatment with caffeine was begun 3 weeks before viral delivery onwards, since we have previously 
reported that a minimum period of 2 weeks is required to allow a metabolic stabilization after beginning the free access to caffeine drinking. ${ }^{29,31}$

\section{Viral vectors production}

Lentiviral vectors encoding human wild-type ataxin-3 (atx3-27Q) or mutant ataxin-3 (atx3-72Q), ${ }^{8}$ were produced in 293T cells with a four-plasmid system, as previously described. $^{32}$ The lentiviral particles were resuspended in $1 \%$ bovine serum albumin in phosphate-buffered saline (PBS). The viral particle content of batches was determined by assessing HIV-1 p24 antigen levels (RETROtek, Gentaur, Paris, France). Viral stocks were stored at $-80^{\circ} \mathrm{C}$ until use.

\section{In vivo injection into the striatum}

Concentrated viral stocks were thawed on ice. After anesthesia of the mice with avertin (12 $\mu \mathrm{L} / g$, i.p.), lentiviral vectors encoding human wild-type (atx3-27Q) or mutant ataxin-3 (atx3-72Q) were stereotaxically injected into the striatum in the following coordinates: anteroposterior: $+0,6 \mathrm{~mm}$; lateral: $\pm 1,8 \mathrm{~mm}$; ventral: $-3,3 \mathrm{~mm}$; tooth bar: 0 , which corresponds to the internal capsule, a large fiber tract passing through the middle of the striatum dividing both dorso-ventral and medial-lateral structures. Wild-type and $A_{2 A} R K O$ mice received $2 \mu \mathrm{L}$ injections of lentivirus (200'000 ng of $\mathrm{p} 24 / \mathrm{mL}$ ) in each hemisphere, administering mutated ataxin-3 (atx3-72Q) in the right hemisphere and control wild type ataxin-3 (atx3-27Q) in the left hemisphere. Different groups of mice were kept in their home cages for different periods ranging from 2, 4, 8 and 12 weeks, before being killed for immunohistochemical analysis of morphological and neurochemical changes in the striatum.

\section{Immunohistochemical procedure}

After an overdose of avertin (2.5x $12 \mu \mathrm{L} / \mathrm{g}$, i.p.), transcardiac perfusion of the mice was performed with PBS followed by fixation with $4 \%$ paraformaldehyde. The brains were then removed and post-fixed in $4 \%$ paraformaldehyde for $24 \mathrm{~h}$ and cryoprotected by incubation in $25 \%$ sucrose/ phosphate buffer for $48 \mathrm{~h}$. The brains were frozen and $25 \mu \mathrm{m}$ coronal sections 
were cut using a cryostat (LEICA CM3050 S, Heidelberg, Germany) at $-21^{\circ} \mathrm{C}$. Slices throughout the entire striatum were collected in anatomical series and stored in 48-well trays as free-floating sections in PBS supplemented with $0.05 \mu \mathrm{M}$ sodium azide. The trays were stored at $4^{\circ} \mathrm{C}$ until immunohistochemical processing.

Sections were processed overnight at $4^{\circ} \mathrm{C}$ with the following primary antibodies: a mouse monoclonal anti-ataxin-3 antibody (1H9; 1:5000; Chemicon, Temecula, CA), a rabbit anti-DARPP-32 antibody (1:1000; Chemicon), or a mouse anti-NeuN antibody (1:1000; Chemicon) followed by $2 h$ incubation at room temperature (RT) with the respective biotinylated secondary antibodies (1:200; Vector Laboratories, Burlingame, CA). Bound antibodies were visualized using the Vectastain ABC kit, with 3,3'-diaminobenzidine tetrahydrochloride (DAB metal concentrate; Pierce, Burlingame, $C A$ ) as substrate.

Triple staining for synaptophysin (rabbit polyclonal, 1:300; Chemicon) and MAP2 (mouse monoclonal AP20, 1:500; Santa Cruz Biotechnology, Santa Cruz, CA), GFAP (rabbit polyclonal, 1:1000; DAKO, Glostrup, Denmark) and CD11b (rat monoclonal 5C6, 1:500; AbD Serotec, Oxford, UK) together with DAPI (Sigma, St. Louis, MO) were performed. Freefloating sections were kept at RT for $2 \mathrm{~h}$ in PBS with $0.1 \%$ Triton $\mathrm{X}-100$ containing $10 \%$ normal goat serum (Gibco-Invitrogen, Barcelona, Spain), then overnight at $4^{\circ} \mathrm{C}$ in blocking solution with the primary antibodies. Sections were washed three times and incubated for $2 \mathrm{~h}$ at RT with the corresponding secondary antibodies coupled to fluorophores goat anti-mouse or goat anti-rabbit or goat anti-rat Alexa Fluor 488 or Alexa Fluor 594 (1:200; Molecular Probes - Invitrogen, Eugene, OR) diluted in the blocking solution. The sections were washed three times and then mounted in mowiol Reagent (Sigma) on microscope slides.

Definition and analysis of protein immunoreactivities were made from the striatal center (site of injection) to the medial-lateral and dorsal-ventral striatal periphery from the needle tract. This disease model is based on the intra-striatal injection of lentivirus, which triggers physiological alterations evolving over time radially. Therefore, comparable striatal sections 
between animals were defined from the site of injection in both rostral and caudal directions, using the needle tract due to the surgical procedure as reference-point.

Staining was visualized using Zeiss Axioskop 2 plus, Zeiss Axiovert 200 or Zeiss LSM 510 Meta imaging microscopes (Carl Zeiss Microimaging, Germany) equipped with AxioCam HR color digital cameras (Carl Zeiss Microimaging) and 5X, 20X, 40X and 63X Plan-Neofluar or a $63 \mathrm{X}$ Plan/Apochromat objectives and using the AxioVision 4.7 software package (Carl Zeiss Microimaging). Quantitative analysis of fluorescence was performed with a semiautomated image-analysis software package (Image $\mathrm{J}$ software, $\mathrm{NIH}, \mathrm{USA}$ ).

\section{Cresyl violet staining}

Coronal sections were pre-mounted and stained with cresyl violet for $45 \mathrm{sec}$, differentiated in $70 \%$ ethanol, dehydrated by passing twice through $95 \%$ ethanol, $100 \%$ ethanol and xylene solutions, and mounted onto microscope slides with Eukitt ${ }^{\circledR}$ (Sigma).

\section{Evaluation of the volume of the DARPP-32 depleted volume and optic density}

The extent of ataxin-3 lesions in the striatum was analyzed by photographing, with a x1.25 objective, 8 sections stained with DARPP-32 per animal $(25 \mu \mathrm{m}$ thick sections at 200 $\mu \mathrm{m}$ intervals), selected so as to obtain complete rostro-caudal sampling of the striatum, and by quantifying the area of the lesion with a semi-automated image-analysis software package (Image $\mathrm{J}$ software). The volume was then estimated with the following formula: volume = $d\left(a_{1}+a_{2}+a_{3} \ldots\right)$, where $d$ is the distance between serial sections $(200 \mu \mathrm{m})$ and $a_{1}+a_{2}+a_{3}$ are DARPP-32-depleted areas for individual serial sections. The average grey value of all pixels measured in the lesioned area was recorded for each depleted area. Results are expressed as index of immunoreactivity of DARPP-32 considering unlesioned striatal area as $100 \%$ immunoreactivity.

\section{Cell counts of ataxin-3 inclusions}

Coronal sections showing complete rostro-caudal sampling (1 of 8 sections) of the striatum were scanned with a $\times 20$ objective. The analyzed areas of the striatum 
encompassed the entire region containing ataxin- 3 inclusions, as revealed by staining with the anti-ataxin-3 antibody. All inclusions were manually counted using a semi-automated image-analysis software package (Image $\mathrm{J}$ software).

\section{Statistical analysis}

Statistical comparisons were performed using either an unpaired Student's $t$ test or one-way analysis of variance (ANOVA) followed by Dunnett's multiple comparison post hoc test. Results are expressed as mean \pm standard error of the mean (SEM). Significance thresholds were set at $p<0.05, p<0.01$ or $p<0.001$, as defined in the text.

\section{Results}

\section{Time course}

We took advantage of using a lentiviral model of MJD to perform a temporal analysis of the relative appearance of different features from early dysfunction to late neuronal loss. Thus, we carried out a time-course study from 2 to 12 weeks upon lentiviral-mediated expression of wild-type and mutant ataxin-3, and we analysed different neuropathological features such as markers of synaptic loss, neuronal dysfunction, neuronal loss, of astrogliosis and microgliosis as well as ataxin-3 inclusions. Figure 1 summarises the temporal evolution of each of these changes, which will be further detailed below.

\section{Neuronal degeneration and loss}

In order to directly test if caffeine and $A_{2 A} R$ inactivation indeed mitigate neurodegeneration, we first analyzed photomicrographs under bright field and then upon cresyl violet staining. Lentiviral-mediated expression of mutant ataxin-3 (LV-atx3-72Q) caused a clear condensation of the internal capsule attributable to striatal tissue shrinkage, which was evident in water-drinking mice at 12 weeks, but absent on bright-field sections of both caffeine-drinking animals and global $A_{2 A} R$ knockout $(K O)$ mice, as well as in the contralateral striatum challenged with wild-type ataxin-3 (LV-atx3-27Q) (Fig 2A). Cresyl violet staining revealed a marked reduction $(p<0.05)$ of the number of degenerated shrunken 
hyperchromatic nuclei in caffeine-drinking mice even though it remained considerably high; the specificity of the effect over $A_{2 A} R$ was confirmed in $A_{2 A} R K O$ animals, which reproduced the alleviation of pathology observed in caffeine-treated animals $(p<0.05$; Fig 2B and E).

To further investigate the neuroprotective effects of caffeine at a late time-point in this model of MJD, we evaluated the immunoreactivity of the neuronal nuclear marker NeuN. A clear loss of NeuN-stained neurons could be seen in the water-drinking group at 12 weeks after mutant ataxin-3 transduction, which was not detectable after wild-type ataxin-3 transduction, and was nearly absent in mutant ataxin-3-expressing animals upon caffeine treatment (Fig 2C).

These data suggest that there is a progression in the degeneration pattern in MJDassociated striatal pathology leading to loss of neuronal markers, which is reduced by chronic caffeine consumption as well as genetically deleting the $A_{2 A} R$.

\section{Neuronal functional modifications}

Previous reports have indicated that striatal neuronal dysfunction may precede degeneration and appearance of clinical symptoms in MJD. ${ }^{33}$ Additionally, DARPP-32 (dopamine and cAMP-regulated phosphoprotein) was previously shown to be a sensitive marker that allows immunohistochemical detection of this early neuronal dysfunction. $8,27,34,35$ Accordingly, DARPP-32 immunohistochemistry revealed a large depleted staining volume of $0.60 \pm 0.12 \mathrm{~mm}^{3}(\mathrm{n}=5)$ at 4 weeks post-injection of lentivirus encoding atx3-72Q in the waterdrinking group whereas caffeine-treated animals exhibited significantly smaller dysfunctional volume $\left(0.21 \pm 0.06 \mathrm{~mm}^{3}(\mathrm{n}=6) ; p<0.05\right)$ at this time-point. No loss of DARPP-32 immunoreactivity was detected upon LV-atx3-27Q injections (Fig 3A upper panel and B).

At higher magnification, analysis of the striatal DARPP-32-depleted area of waterdrinking animals at 12 weeks after injection of lentiviral vectors encoding mutant ataxin-3 revealed that the DARPP-32-stained cell bodies and the corresponding tissue (internal capsule) were no longer present, originating a collapse of the tissue, which joins together 
fiber patches (see arrows in Fig 3A lower panel). This fiber accumulation presumably results from neuronal degeneration, which was not observed in the caffeine-treated or in $A_{2 A} R K O$ mice challenged with mutant ataxin-3. Density analysis of DARPP-32 immunoreactivity (Table 1) showed a significant preservation of this marker in caffeine-treated $(p<0.01)$ and $\mathrm{A}_{2 \mathrm{~A}} \mathrm{R}$ KO groups $(p<0.05)$ at 12 weeks post-injection of lentivirus encoding atx3-72Q, as compared to the respective water-drinking groups.

These data suggest that both chronic caffeine consumption as well as the genetic deletion of $A_{2 A} R$ is able to reduce neuronal dysfunction in MJD.

\section{Astrogliosis and microgliosis}

Interestingly, the mutant ataxin-3-induced loss of NeuN immunoreactivity was accompanied by a local increase of GFAP immunoreactivity suggestive of astrocytic activation, which was nearly absent in caffeine-treated animals (Fig 2D). Since reactive gliosis is widely accepted to contribute to chronic neurodegenerative diseases, we further investigated if the blockade of $A_{2 A} R$ was able to prevent the increase of gliosis associated with MJD pathogenesis at an early time-point. The injection of lentiviral vectors encoding mutant ataxin-3 (LV-atx3-72Q) triggered a robust increase of GFAP immunoreactivity at 4 weeks when compared with the contralateral striatum challenged with wild-type ataxin-3 (LVatx3-27Q) (Fig 4A). Notably, this GFAP immunoreactivity triggered by expression of mutated ataxin-3 was observed as early as 2 weeks (Fig 4B). Additionally, strong immunoreactivity for the microglial protein, cluster of differentiation molecule B11 (CD11b), was found at 4 weeks revealing microglial recruitment (Fig 4C), which was significantly and robustly reduced in caffeine-treated animals. This clearly establishes the presence of reactive gliosis in the striatum in this genetic model of MJD.

Importantly, treatment with caffeine $(1 \mathrm{~g} / \mathrm{L})$, prevented both the astrogliosis (Fig 4A and B) and the putative microgliosis (Fig 4C) triggered by mutant ataxin-3 indicating that chronic caffeine consumption can prevent reactive gliosis associated with MJD. 


\section{Nuclear inclusions of mutant ataxin-3}

Since microglia is well established to play a role in the seeding (through enhanced inflammatory status) and processing (through phagocytosis) of protein aggregates and the presence of ataxin-3 aggregates is one hallmark of MJD, we next tested the impact of caffeine and $A_{2 A} R$ inactivation on the aggregation pattern of ataxin-3 by immunohistochemical staining. No aggregation was seen upon LV-atx3-27Q injection (Fig $5 \mathrm{~A}$ ) while a significant increase in the total number of mutant ataxin-3 nuclear inclusions was observed at 8 weeks post-injection of lentiviral vectors encoding mutant ataxin- 3 in both caffeine-drinking wild type mice $(p<0.05)$ as well as in $A_{2 A} R$ KO animals $(p<0.05)$ when compared to the respective water-drinking groups (Fig 5B and $\mathrm{C}$ ). Interestingly, we previously demonstrated that at 8 weeks after lentiviral transduction there were 4 times higher striatal levels of mutant ataxin- 3 than those of endogenous ataxin- $3,{ }^{8}$ although the levels were similar 4 weeks after lentiviral administration. This observation that caffeine or $A_{2 A} R$ inactivation enhance even more the number of ataxin-3 inclusions while decreasing neurodegeneration is in agreement with the scenario that these inclusions may be a way to sequester the soluble and noxious forms of ataxin-3.

\section{Synaptotoxicity}

There is accumulating evidence that one of the earliest features of neurodegenerative diseases is the dysfunction and loss of synapses. ${ }^{24,36}$ Also, $A_{2 A} R$ are synaptic receptors, ${ }^{37}$ and $A_{2 A} R$ blockade efficiently normalizes synaptic function and prevents synaptotoxicity in different animal models of brain diseases. ${ }^{22}$ Since synaptotoxicity has not been explored in models of MJD, we investigated whether synaptotoxicity was present in our lentiviral model of MJD and if this feature indeed preceded the appearance of ataxin-3 inclusions, neuronal dysfunction and overt neurodegeneration. This was carried out by immunohistochemical evaluation of two synaptic markers, synaptophysin (pre-synaptic protein) and microtubuleassociated protein 2 (MAP-2, dendritic protein). 
Density analysis of synaptophysin and MAP-2 immunoreactivities at 2 weeks postinjection (Table 2 and Fig 6 ) clearly showed a significant loss of both markers $(p<0.001)$ upon mutant ataxin-3 expression. No such early loss of either markers was observed in caffeine-drinking mice challenged with atx3-72Q, i.e. 2 weeks after insult or upon expression of wild-type ataxin-3 (data not shown). These data provide the first evidence that mutant ataxin-3 induces an early synaptotoxicity in the striatum, which is prevented by chronic caffeine consumption.

\section{Discussion}

In the present study, we carried out a temporal analysis of different features of brain dysfunction and damage in a genetic model of MJD and tested the impact of caffeine and adenosine $A_{2 A} R\left(A_{2 A} R\right)$ blockade thereupon. We provide the first evidence showing that: i) synaptotoxicity and gliosis in the striatum are early events predating neurodegeneration; ii) pharmacological and genetic manipulation of adenosine $A_{2 A} R$ can delay MJD-associated striatal pathology.

Consistent with previous reports, ${ }^{8,13,14}$ lentiviral-mediated overexpression of mutant ataxin-3 in the mouse brain induced a clear neuronal dysfunction typified by loss of DARPP32 immunoreactivity, and overt neurodegeneration, accompanied by an increased number of ataxin-3 inclusions. The present study also revealed two novel morphological features in this model of MJD, namely the loss of synaptic markers (synaptotoxicity) and reactive gliosis. Synaptotoxicity is in line with suggestions of axonal degeneration assessed by $\mathrm{MRI},{ }^{38}$ and loss of dopaminergic terminals assessed by PET in MJD patients, ${ }^{11}$ and with the presence of axonal inclusions in the human patient's brains, ${ }^{39}$ as well as with the observed impact of mutant ataxin-3 on the cerebellar mRNA expression of proteins involved in synaptic transmission. ${ }^{40}$ Notably, the present time course study provides direct evidence that this synaptotoxicity might be an early, hitherto unrecognised, feature of MJD. This is in notable agreement with the observations that another polyglutamine-related disease, namely Huntington's disease is characterized by early changes in synapses, ${ }^{41,42}$ to such an extent 
that it has been proposed that Huntington's disease might actually be a synaptopathy. ${ }^{43}$ Actually, it is worth noting that synaptotoxicity seems to be an early feature of different other neurodegenerative and neuropsychiatric diseases, strengthening the crucial role of synaptic impairment in the initiation of brain disorders. ${ }^{22,36,44}$ Since there is evidence that ataxin-3 is also located in axons and dendrites, ${ }^{45}$ the present observation that synaptic changes are an early feature of MJD opens a new area of research on the putative role of ataxin-3 in the control of synaptic function and damage. In this context, the recent report that excitatory synaptic transmission can control the aggregation of mutant ataxin-3 adds a further dimension to the relation between MJD and synaptic activity. ${ }^{26}$

The present time course study of striatal changes in this MJD model also revealed another under-appreciated morphological feature that predated neuronal dysfunction and damage, namely reactive gliosis. There has been episodic reports of astrogliosis and microgliosis, typified by changes in astrocytes and microglia morphology both in patients, ${ }^{46}$ and in transgenic models of $\mathrm{MJD},{ }^{47}$ as well as increased expression of cytokines and proinflammatory chemokines, which are compatible with mutant ataxin 3-induced changes in brain inflammatory mediators. ${ }^{48}$ However, in keeping with the fact that our lentiviral-mouse model resulted in an overexpression of mutant ataxin-3 even in comparable levels to the endogenous form, the present report provides evidence that reactive astrogliosis might be an early feature in MJD, which is particularly relevant in view of the surge of interest in the role of non-neuronal brain cells in the aetiology of neurodegenerative disorders. ${ }^{49}$ A putative role of glial cells in MJD is further heralded by evidence of the presence of ataxin-3 in glial cells. ${ }^{12,50}$ Again, this observation should open a novel area of research fostering a better understanding of the role of ataxin-3 in astrocytes and on the consequences of astrocytic adaptation upon accumulation of mutated ataxin-3.

Thus, the present time-course exploration of neuropathological features associated with this genetic model of MJD identifies synaptotoxicity and astrogliosis as precocious modifications followed by microgliosis and neuronal dysfunction, appearance of NIls and 
overt neuronal damage. This time course is in general agreement with the recognition that synaptotoxicity and astrocytic-related metabolic imbalance are amongst the most precocious modifications in different neurodegenerative disorders and that neuroinflammation, previously implicated in MJD, ${ }^{48}$ may be a candidate process to mediate the spreading and amplification of damage until overt neuronal dysfunction and damage can be recorded. ${ }^{24,36,49}$

The second prominent conclusion of this study is the demonstration that the chronic consumption of a reasonable dose of caffeine compatible to a significant blockade of adenosine effects on $A_{2 A}$ (most potent) and $A_{1}$ receptors ${ }^{51}$ or the genetic elimination of $A_{2 A} R$ mitigated the striatal neuropathological modifications caused by the expression of mutated ataxin-3. This is in agreement with the ability of $A_{2 A} R$, mainly targeted by chronic caffeine consumption, ${ }^{22,52}$ to afford neuroprotection against different neurodegenerative disorders, namely Alzheimer's, Parkinson's or Huntington's disease. ${ }^{22,25,53}$ Notably, most of the compartments that we now showed to be affected in this genetic model of MJD are effectively normalized by $A_{2 A} R$ blockade in chronic brain diseases: thus, $A_{2 A} R$ blockade prevents synaptotoxicity, ${ }^{54,55}$ in accordance with the enrichment of $A_{2 A} R$ in synapses, ${ }^{37}$ and also controls astrogliosis, ${ }^{56-58}$ and microgliosis, ${ }^{59}$ striatal neurodegeneration, ${ }^{60}$ and neuronal death. ${ }^{23,61}$ Accordingly, in our genetic model of MJD, chronic caffeine consumption or genetic deletion of $A_{2 A} R$ abrogated the loss of synaptic markers, prevented astrogliosis and microglia activation, reduced cell injury and striatal degeneration rescuing its normal cytoarchitecture. Therefore, this provides the first evidence that the manipulation of a neuromodulation system operated by $A_{2 A} R$ is effective in controlling the initial cascade of events triggered by the pathogenic ataxin-3 protein (synaptotoxicity and gliosis) that culminate in a reduced neuronal degeneration.

It is worth noting that this general neuroprotection afforded by caffeine and $A_{2 A} R$ blockade is accompanied by an accumulation of ataxin- 3 aggregates into intracellular nuclear inclusions. The inverse correlation between the impact of caffeine and $A_{2 A} R$ blockade on mutant ataxin-3-induced NIls and neuropathology strongly suggests that the aggregation of 
ataxin-3 could correspond to a cellular defensive mechanism against soluble, more toxic species, ${ }^{62-65}$ rather than being the main cause of degeneration. Therefore, although it cannot be excluded that this might result from a better neuronal survival in treated animals, we hypothesized that aggregates may be neuroprotective. Nevertheless, the neuroprotection conveyed by caffeine and $A_{2 A} R$ blockade might be of limited duration since the number of pycnotic nuclei remained relatively high.

The present exploration of the time course of MJD-associated neuropathological features and its modification by $A_{2 A} R$ provides a novel insight into the neuropathology of MJD but does not explore the underlying mechanistic processes. Ataxin-3 is a polyubiquitinbinding protein whose physiological function has been linked to de-ubiquitination, ${ }^{66-69}$ and MJD is argued to result from a toxic gain of function of mutant ataxin-3. In keeping with our proposal that synaptotoxicity might be a precocious modification in MJD, several studies highlight the importance of the ubiquitin-proteosome system (UPS) in synapses, ${ }^{70,71}$ namely in presynaptic terminals, ${ }^{72,73}$ where it is affected in other polyQ neurological diseases; ${ }^{74}$ furthermore, there is preliminary evidence that $A_{2 A} R s$ directly bind to UPS components, ${ }^{75}$ and control the UPS activity, ${ }^{76}$ paving the way for a putative direct control by $A_{2 A} R$ of synaptic UPS. The proposal that the ability of $A_{2 A} R s$ to control another polyQ neurological disorder (Huntington's disease) depends on the control of glutamatergic transmission prompts an alternative mechanism by which the $A_{2 A} R$-mediated control of the initial event in MJD might be related to the ability of $A_{2 A} R$ to control abnormal glutamatergic transmission through direct synaptic effects, ${ }^{77}$ or indirectly through control of astrocytic glutamate uptake. ${ }^{78,79}$ Thus, albeit the molecular mechanism of $A_{2 A} R$-mediated control of MJD remains to be determined, the present study provides new clues for particular compartments where such mechanisms should be explored.

In conclusion, the present study provides a novel insight into the pathology of MJD bringing to the centre stage synaptotoxicity and gliosis as precocious events in MJD. Furthermore, it provides the first realistic and safe promising life style prophylactic strategy to 
delay the onset of this inherited disorder, based on the consumption of caffeine. Finally, it provides the first suggestion that $A_{2 A} R$ might be a novel therapeutic target to interfere with the inexorable evolution of this neurodegenerative disease.

\section{Acknowledgements}

This work was supported by Fundação para a Ciência e a Tecnologia (FCT), SAUFCF/70384/2006, SAU-NEU/099307/2008, SAU-NEU/108668/2008, The Richard Chin and Lily Lock Machado Joseph disease Research Fund and the Association Française Contre les Myopathies (AFM). N.G. and A.T.S. were supported by FCT fellowships $\mathrm{BD} / 33186 / 2007$ and BD/38636/2007.

\section{References}

1. Koshy BT, Zoghbi HY. The CAG/polyglutamine tract diseases: gene products and molecular pathogenesis. Brain Pathol. 1997;7:927-942

2. Ranum LP, Lundgren JK, Schut LJ et al. Spinocerebellar ataxia type 1 and MachadoJoseph disease: incidence of CAG expansions among adult-onset ataxia patients from 311 families with dominant, recessive, or sporadic ataxia. Am J Hum Genet. 1995;57:603608

3. Durr A, Stevanin G, Cancel G et al. Spinocerebellar ataxia 3 and Machado-Joseph disease: clinical, molecular, and neuropathological features. Ann Neurol. 1996;39:490499

4. Kawaguchi $\mathrm{Y}$, Okamoto $\mathrm{T}$, Taniwaki $\mathrm{M}$ et al. CAG expansions in a novel gene for Machado-Joseph disease at chromosome 14q32.1. Nat Genet. 1994;8:221-228

5. Gwinn-Hardy K, Singleton A, O'Suilleabhain $P$ et al. Spinocerebellar ataxia type 3 phenotypically resembling parkinson disease in a black family. Arch Neurol. 2001;58:296299 
6. Taroni F, DiDonato S. Pathways to motor incoordination: the inherited ataxias. Nat Rev Neurosci. 2004;5:641-655

7. Sudarsky L, Coutinho P. Machado-Joseph disease. Clin Neurosci. 1995;3:17-22

8. Alves S, Regulier E, Nascimento-Ferreira I et al. Striatal and nigral pathology in a lentiviral rat model of Machado-Joseph disease. Hum Mol Genet. 2008;17:2071-2083

9. Klockgether T, Skalej M, Wedekind D et al. Autosomal dominant cerebellar ataxia type I. MRI-based volumetry of posterior fossa structures and basal ganglia in spinocerebellar ataxia types 1, 2 and 3. Brain. 1998;121:1687-1693

10. Taniwaki T, Sakai T, Kobayashi T et al. Positron emission tomography (PET) in MachadoJoseph disease. J Neurol Sci. 1997;145:63-67

11. Wullner $U$, Reimold $M$, Abele $M$ et al. Dopamine transporter positron emission tomography in spinocerebellar ataxias type 1, 2, 3, and 6. Arch Neurol. 2005;62:12801285

12. Paulson HL, Perez MK, Trottier Y et al. Intranuclear inclusions of expanded polyglutamine protein in spinocerebellar ataxia type 3. Neuron. 1997;19:333-344

13. Bichelmeier $\mathrm{U}$, Schmidt $\mathrm{T}$, Hubener $\mathrm{J}$ et al. Nuclear localization of ataxin-3 is required for the manifestation of symptoms in SCA3: in vivo evidence. J Neurosci. 2007;27:7418-7428

14. Goti D, Katzen SM, Mez J et al. A mutant ataxin-3 putative-cleavage fragment in brains of Machado-Joseph disease patients and transgenic mice is cytotoxic above a critical concentration. J Neurosci. 2004;24:10266-10279

15. Cemal CK, Carroll CJ, Lawrence $\mathrm{L}$ et al. YAC transgenic mice carrying pathological alleles of the MJD1 locus exhibit a mild and slowly progressive cerebellar deficit. Hum Mol Genet. 2002;11:1075-1094

16. Andrews TC, Weeks RA, Turjanski $\mathrm{N}$ et al. Huntington's disease progression. PET and clinical observations. Brain. 1999;122:2353-2363 
17. Li H, Li SH, Yu ZX et al. Huntingtin aggregate-associated axonal degeneration is an early pathological event in Huntington's disease mice. J Neurosci. 2001;21:8473-8481

18. Bantubungi K, Jacquard C, Greco A et al. Minocycline in phenotypic models of Huntington's disease. Neurobiol Dis. 2005;18:206-217

19. Aktas O, Smorodchenko A, Brocke $S$ et al. Neuronal damage in autoimmune neuroinflammation mediated by the death ligand TRAIL. Neuron. 2005;46:421-432

20. Gao HM, Kotzbauer PT, Uryu K et al. Neuroinflammation and oxidation/nitration of $\alpha$ synuclein linked to dopaminergic neurodegeneration. J Neurosci. 2008;28:7687-7698

21. Lee JW, Lee YK, Yuk DY et al. Neuro-inflammation induced by lipopolysaccharide causes cognitive impairment through enhancement of beta-amyloid generation. J Neuroinflammation. 2008;5:37

22. Cunha RA, Agostinho PM. Chronic caffeine consumption prevents memory disturbance in different animal models of memory decline. J Alzheimers Dis. 2010;20 Suppl 1:S95-S116

23. Chen JF, Sonsalla PK, Pedata $F$ et al. Adenosine $A_{2 A}$ receptors and brain injury: broad spectrum of neuroprotection, multifaceted actions and "fine tuning" modulation. Prog Neurobiol. 2007;83:310-331

24. Gomes CV, Kaster MP, Tome AR et al. Adenosine receptors and brain diseases: neuroprotection and neurodegeneration. Biochim Biophys Acta. 2011;1808:1380-1399

25. Popoli P, Blum D, Martire A et al. Functions, dysfunctions and possible therapeutic relevance of adenosine $A_{2 A}$ receptors in Huntington's disease. Prog Neurobiol. $2007 ; 81: 331-348$

26. Koch P, Breuer P, Peitz M et al. Excitation-induced ataxin-3 aggregation in neurons from patients with Machado-Joseph disease. Nature. 2011;480:543-546

27. Simões AT, Gonçalves N, Koeppen A et al. Calpastatin-mediated inhibition of calpains in the mouse brain prevents mutant ataxin-3 proteolysis, nuclear localization and aggregation, relieving Machado-Joseph disease. Brain. 2012;135:2428-2439 
28. Chen JF, Huang $Z$, Ma J et al. $A_{2 A}$ adenosine receptor deficiency attenuates brain injury induced by transient focal ischemia in mice. J Neurosci. 1999;19:9192-9200

29. Duarte JM, Agostinho PM, Carvalho RA, Cunha RA. Caffeine consumption prevents diabetes-induced memory impairment and synaptotoxicity in the hippocampus of NONcZNO10/LTJ mice. PLoS One. 2012;7:e21899

30. Costenla AR, Cunha RA, de Mendonca A. Caffeine, adenosine receptors, and synaptic plasticity. J Alzheimers Dis. 2010;20 Suppl 1:S25-S34

31. Duarte JM, Carvalho RA, Cunha RA, Gruetter R. Caffeine consumption attenuates neurochemical modifications in the hippocampus of streptozotocin-induced diabetic rats. J Neurochem. 2009;111:368-379

32. de Almeida LP, Zala D, Aebischer P, Deglon N. Neuroprotective effect of a CNTFexpressing lentiviral vector in the quinolinic acid rat model of Huntington's disease. Neurobiol Dis. 2001;8:433-446

33. Yen TC, Tzen KY, Chen MC et al. Dopamine transporter concentration is reduced in asymptomatic Machado-Joseph disease gene carriers. J Nucl Med. 2002;43:153-159

34. de Almeida LP, Ross CA, Zala D et al. Lentiviral-mediated delivery of mutant huntingtin in the striatum of rats induces a selective neuropathology modulated by polyglutamine repeat size, huntingtin expression levels, and protein length. J Neurosci. 2002;22:34733483

35. Cyr M, Beaulieu JM, Laakso A et al. Sustained elevation of extracellular dopamine causes motor dysfunction and selective degeneration of striatal GABAergic neurons. Proc Natl Acad Sci U S A. 2003;100:11035-11040

36. Coleman P, Federoff H, Kurlan R. A focus on the synapse for neuroprotection in Alzheimer disease and other dementias. Neurology. 2004;63:1155-1162

37. Rebola N, Canas PM, Oliveira CR, Cunha RA. Different synaptic and subsynaptic localization of adenosine $A_{2 A}$ receptors in the hippocampus and striatum of the rat. Neuroscience. 2005;132:893-903 
38. D'Abreu A, Franca M, Jr., Appenzeller $S$ et al. Axonal dysfunction in the deep white matter in Machado-Joseph disease. J Neuroimaging. 2009;19:9-12

39. Seidel K, den Dunnen WF, Schultz C et al. Axonal inclusions in spinocerebellar ataxia type 3. Acta Neuropathol. 2010;120:449-460

40. Chou AH, Chen SY, Yeh TH et al. HDAC inhibitor sodium butyrate reverses transcriptional downregulation and ameliorates ataxic symptoms in a transgenic mouse model of SCA3. Neurobiol Dis. 2011;41:481-488

41. DiProspero NA, Chen EY, Charles V et al. Early changes in Huntington's disease patient brains involve alterations in cytoskeletal and synaptic elements. J Neurocytol. $2004 ; 33: 517-533$

42. Smith R, Klein P, Koc-Schmitz $\mathrm{Y}$ et al. Loss of SNAP-25 and rabphilin 3a in sensorymotor cortex in Huntington's disease. J Neurochem. 2007;103:115-123

43. Li JY, Plomann M, Brundin P. Huntington's disease: a synaptopathy? Trends Mol Med. $2003 ; 9: 414-420$

44. Wishart TM, Parson SH, Gillingwater TH. Synaptic vulnerability in neurodegenerative disease. J Neuropathol Exp Neurol. 2006;65:733-739

45. Trottier Y, Cancel G, An-Gourfinkel I et al. Heterogeneous intracellular localization and expression of ataxin-3. Neurobiol Dis. 1998;5:335-347

46. Horimoto $\mathrm{Y}$, Matsumoto $\mathrm{M}$, Akatsu $\mathrm{H}$ et al. Longitudinal study on MRI intensity changes of Machado-Joseph disease: correlation between MRI findings and neuropathological changes. J Neurol. 2011;258:1657-1664

47. Silva-Fernandes A, Costa Mdo C, Duarte-Silva S et al. Motor uncoordination and neuropathology in a transgenic mouse model of Machado-Joseph disease lacking intranuclear inclusions and ataxin-3 cleavage products. Neurobiol Dis. 2010;40:163-176

48. Evert BO, Vogt IR, Kindermann C et al. Inflammatory genes are upregulated in expanded ataxin-3-expressing cell lines and spinocerebellar ataxia type 3 brains. J Neurosci. $2001 ; 21: 5389-5396$ 
49. Lobsiger CS, Cleveland DW. Glial cells as intrinsic components of non-cell-autonomous neurodegenerative disease. Nat Neurosci. 2007;10:1355-1360

50. Wang G, Ide $\mathrm{K}$, Nukina $\mathrm{N}$ et al. Machado-Joseph disease gene product identified in lymphocytes and brain. Biochem Biophys Res Commun. 1997;233:476-479

51. Fredholm BB, Battig $\mathrm{K}$, Holmen $\mathrm{J}$ et al. Actions of caffeine in the brain with special reference to factors that contribute to its widespread use. Pharmacol Rev. 1999;51:83133

52. Ferré S. An update on the mechanisms of the psychostimulant effects of caffeine. J Neurochem. 2008;105:1067-1079

53. Schwarzschild MA, Agnati L, Fuxe $K$ et al. Targeting adenosine $A_{2 A}$ receptors in Parkinson's disease. Trends Neurosci. 2006;29:647-654

54. Canas PM, Porciuncula LO, Cunha GM et al. Adenosine $A_{2 A}$ receptor blockade prevents synaptotoxicity and memory dysfunction caused by beta-amyloid peptides via p38 mitogen-activated protein kinase pathway. J Neurosci. 2009;29:14741-14751

55. Silva CG, Porciuncula LO, Canas PM et al. Blockade of adenosine $\mathrm{A}_{2 \mathrm{~A}}$ receptors prevents staurosporine-induced apoptosis of rat hippocampal neurons. Neurobiol Dis. 2007;27:182-189

56. Brambilla $R$, Cottini $L$, Fumagalli $M$ et al. Blockade of $A_{2 A}$ adenosine receptors prevents basic fibroblast growth factor-induced reactive astrogliosis in rat striatal primary astrocytes. Glia. 2003;43:190-194

57. Minghetti L, Greco A, Potenza RL et al. Effects of the adenosine $A_{2 A}$ receptor antagonist $\mathrm{SCH} 58621$ on cyclooxygenase-2 expression, glial activation, and brain-derived neurotrophic factor availability in a rat model of striatal neurodegeneration. $\mathrm{J}$ Neuropathol Exp Neurol. 2007;66:363-371

58. Yu L, Shen HY, Coelho JE et al. Adenosine $A_{2 A}$ receptor antagonists exert motor and neuroprotective effects by distinct cellular mechanisms. Ann Neurol. 2008;63:338-346 
59. Rebola $N$, Simões $A P$, Canas $P M$ et al. Adenosine $A_{2 A}$ receptors control neuroinflammation and consequent hippocampal neuronal dysfunction. J Neurochem. $2011 ; 117: 100-111$

60. Schiffmann SN, Fisone G, Moresco $R$ et al. Adenosine $A_{2 A}$ receptors and basal ganglia physiology. Prog Neurobiol. 2007;83:277-292

61. Cunha RA. Neuroprotection by adenosine in the brain: From $A_{1}$ receptor activation to $A_{2 A}$ receptor blockade. Purinergic Signal. 2005;1:111-134

62. Arrasate M, Mitra S, Schweitzer ES et al. Inclusion body formation reduces levels of mutant huntingtin and the risk of neuronal death. Nature. 2004;431:805-810

63. Saudou F, Finkbeiner S, Devys D, Greenberg ME. Huntingtin acts in the nucleus to induce apoptosis but death does not correlate with the formation of intranuclear inclusions. Cell. 1998;95:55-66

64. Takahashi T, Kikuchi S, Katada S et al. Soluble polyglutamine oligomers formed prior to inclusion body formation are cytotoxic. Hum Mol Genet. 2008;17:345-356

65. Taylor JP, Tanaka F, Robitschek $\mathrm{J}$ et al. Aggresomes protect cells by enhancing the degradation of toxic polyglutamine-containing protein. Hum Mol Genet. 2003;12:749-757

66. Burnett B, Li F, Pittman RN. The polyglutamine neurodegenerative protein ataxin-3 binds polyubiquitylated proteins and has ubiquitin protease activity. Hum Mol Genet. 2003;12:3195-3205

67. Doss-Pepe EW, Stenroos ES, Johnson WG, Madura K. Ataxin-3 interactions with rad23 and valosin-containing protein and its associations with ubiquitin chains and the proteasome are consistent with a role in ubiquitin-mediated proteolysis. Mol Cell Biol. 2003;23:6469-6483

68. Kuhlbrodt K, Janiesch PC, Kevei E et al. The Machado-Joseph disease deubiquitylase ATX-3 couples longevity and proteostasis. Nat Cell Biol. 2011;13:273-281 
69. Warrick JM, Morabito LM, Bilen J et al. Ataxin-3 suppresses polyglutamine neurodegeneration in Drosophila by a ubiquitin-associated mechanism. Mol Cell. 2005;18:37-48

70. Cajigas IJ, Will T, Schuman EM. Protein homeostasis and synaptic plasticity. EMBO J. 2010;29:2746-2752

71. DiAntonio A, Hicke L. Ubiquitin-dependent regulation of the synapse. Annu Rev Neurosci. 2004;27:223-246

72. Jiang $\mathrm{X}$, Litkowski PE, Taylor AA et al. A role for the ubiquitin-proteasome system in activity-dependent presynaptic silencing. J Neurosci. 2010;30:1798-1809

73. Rinetti GV, Schweizer FE. Ubiquitination acutely regulates presynaptic neurotransmitter release in mammalian neurons. J Neurosci. 2010;30:3157-3166

74. Wang J, Wang CE, Orr A et al. Impaired ubiquitin-proteasome system activity in the synapses of Huntington's disease mice. J Cell Biol. 2008;180:1177-1189

75. Milojevic T, Reiterer V, Stefan E et al. The ubiquitin-specific protease Usp4 regulates the cell surface level of the $A_{2 A}$ receptor. Mol Pharmacol. 2006;69:1083-1094

76. Chiang MC, Chen HM, Lai $\mathrm{HL}$ et al. The $\mathrm{A}_{2 \mathrm{~A}}$ adenosine receptor rescues the urea cycle deficiency of Huntington's disease by enhancing the activity of the ubiquitin-proteasome system. Hum Mol Genet. 2009;18:2929-2942

77. Rebola N, Lujan R, Cunha RA, Mulle C. Adenosine $A_{2 A}$ receptors are essential for longterm potentiation of NMDA-EPSCs at hippocampal mossy fiber synapses. Neuron. $2008 ; 57: 121-134$

78. Matos $M$, Augusto $E$, Santos-Rodrigues $A D$ et al. Adenosine $A_{2 A}$ receptors modulate glutamate uptake in cultured astrocytes and gliosomes. Glia. 2012;60:702-716

79. Nishizaki T, Nagai K, Nomura $\mathrm{T}$ et al. A new neuromodulatory pathway with a glial contribution mediated via $\mathrm{A}_{2 a}$ adenosine receptors. Glia. 2002;39:133-147 


\section{Figure Legends and Tables}

Figure 1: Summary of the time-course of appearance of different morphological features in a lentiviral-based model of Machado-Joseph disease. Loss of synaptic markers and astrogliosis were amongst the most precocious morphological alteration, closely followed by microgliosis and neuronal dysfunction as well as increases of the number of ataxin-3 inclusions, whereas overt neuronal damage occurred later in the development of the disease. Microgliosis would probably be found at later time-points, however, that was not evaluated.

Figure 2: $\underline{\text { Caffeine treatment or } A_{2 A}} \underline{R \text { genetic depletion decreased cell injury and striatal }}$ degeneration. Representative bright-field photomicrographs and immunohistochemical stainings from around the injection site area at 12 weeks post-injection of the viral vectors encoding wild-type or mutant ataxin-3. (A) Coalescence of the internal capsule of the striatum was neither seen in the caffeine-treated group nor in the $A_{2 A} R$ KO animals. (B) Cresyl violet-stained sections showed a significant reduction of the number of striatal condensated nuclei upon mutant ataxin-3 transduction in the caffeine-treated group as well as in the $A_{2 A} R$ KO group relative to their respective water-drinking groups, as quantified in panel E. (C) A nearly absent loss of neuronal nuclei (NeuN) staining immunoreactivity was seen in the caffeine-treated group as well as in the $A_{2 A} R$ KO group. (D) GFAP immunoreactivity showed an increased accumulation of astrocytes (green) replacing neurons after injection of mutant ataxin-3, which was prevented by caffeine consumption. No morphological modifications were detected upon LV-atx3-27Q injections. Statistical Significance was evaluated with Student's $t$ test $\left({ }^{*} p<0.05\right)$ comparing both caffeine-drinking and $A_{2 A} R K O$ groups with their respective water-drinking wild-type groups upon LV-atx3-72Q injections.

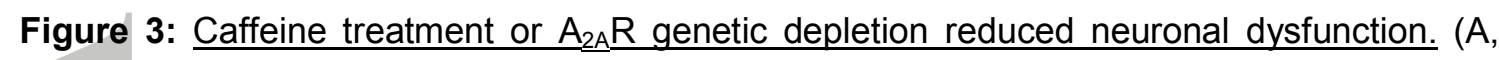
upper panel) A large DARPP-32 depleted volume was observed 4 weeks after injection of the viral vectors encoding mutant ataxin-3 in the water-drinking group whereas caffeinedrinking mice exhibited a much smaller lesion area at this time-point. This is quantified in 
panel $B$ as depleted volume of DARPP-32 staining. No loss of DARPP-32 staining was observed upon LV-atx3-27Q injections. (A, lower panel) At higher magnification, 12 weeks exposure to mutated ataxin-3 revealed a clear condensation of the internal capsule, which joined together fiber patches (arrows). This fiber accumulation was not observed in the caffeine-treated animals or in $A_{2 A} R K O$ mice. Statistical significance was evaluated with Student's $t$ test $\left({ }^{*} p<0.05\right)$ comparing caffeine-treated $(\mathrm{n}=6)$ with water-drinking animals $(n=5)$.

Figure 4: $\underline{\text { Caffeine treatment decreased MJD-associated astroglial activation and prevented }}$ microglia recruitment. (A) Quantification analysis of GFAP immunoreactivity at 4 weeks postinjection of the viral vectors encoding wild-type or mutant ataxin-3. Caffeine abrogated astrocytic activation $\left({ }^{\#} p<0.01\right)$ to the levels induced by wild-type ataxin-3 (internal control). (B) Caffeine consumption attenuated the mutant ataxin-3-induced activation of astrocytes, gauged by enhanced immunoreactivity of Glial Fibrillary Acidic Protein (GFAP, green) at an earlier time point: 2 weeks. (C) No immunoreactivity for activated microglia, CD11b (Ab 5C6, green), was observed 4 weeks after insult in the caffeine-treated group whereas it was present in water-drinking mice. Statistical significance was evaluated with Student's $t$ test $\left({ }^{* *} p<0.01\right)$ comparing mutant ataxin-3 striatal hemisphere with the contralateral wild-type ataxin-3 hemisphere, and caffeine-drinking with water-drinking groups.

Figure 5: $\underline{\text { Caffeine treatment or } A_{2 A} \underline{R} \text { genetic depletion significantly increased the total }}$ number of mutant ataxin-3 inclusions. (A) Using anti-ataxin-3 antibody (Ab 1H9), no nuclear inclusions of ataxin- 3 were found on both wild-type and $A_{2 A} R K O$ animals upon expression of wild-type ataxin-3. (B) A significant increase in the number of mutant ataxin-3 inclusions was observed 8 weeks post-injection of lentiviral vectors encoding mutant ataxin-3 either in caffeine-treated $(n=5)$ or $A_{2 A} R K O(n=5)$ mice compared to the respective water-drinking wildtype animals transduced with mutant ataxin-3 $(B, \mathrm{n}=7 ; E, \mathrm{n}=4)$, as quantified in panel $C$. Statistical significance was evaluated with Student's $t$ test $\left({ }^{*} p<0.05\right)$ comparing caffeinetreated and $A_{2 A} R$ inactivated animals with their respective water-drinking control groups. 
Figure 6: Synaptotoxicity precedes the neurodegeneration process of MJD. Detection of nerve terminals with synaptic markers: synaptophysin (red) and microtubule-associated protein 2 - MAP-2 (Ab AP20, green). No loss of synapses is observed in the group that was given caffeine whereas loss of synaptophysin and MAP-2 immunoreactivity was observed in the non-treated group 2 weeks post-injection of the lentiviral vectors encoding mutant ataxin3.

Table 1: DARPP-32 intensity index

\begin{tabular}{|cc|cc|}
\hline $100 \%$ IR & $100.0 \pm 3.5$ & $100 \%$ IR & $100.0 \pm 4.1$ \\
\hline $\mathrm{H}_{2} \mathrm{O}$ & $43.0 \pm 4.1$ & $\mathrm{H}_{2} \mathrm{O}$ & $39.1 \pm 3.1$ \\
\hline Caffeine & $70.0 \pm 2.2^{* *}$ & $\mathbf{A}_{2 \mathrm{~A}} \mathrm{R} \mathrm{KO}$ & $54.5 \pm 2.2^{*}$ \\
\hline
\end{tabular}

Density analysis of DARPP-32 immunoreactivity 12 weeks after mutant ataxin-3 transduction. Caffeine treatment as well as genetic inactivation of $A_{2 A} R$ significantly reduced the loss of this marker $\left({ }^{* *} p<0.01 ;{ }^{*} p<0.05\right.$, respectively).

Note. Data are expressed as indexes of immunoreactivity of the affected striatal regions relative to their corresponding peripheral non-affected striatum (100\% IR) [mean \pm SEM].

Table 2: Synaptophysin and MAP-2 intensity indexes

\begin{tabular}{|c|c|c|}
\hline & Synaptophysin & MAP-2 \\
\hline $\mathbf{1 0 0 \%}$ IR & $100.0 \pm 3.5$ & $100.0 \pm 2.6$ \\
\hline $\mathbf{H}_{\mathbf{2}} \mathbf{O}$ & $65.5 \pm 4.6^{\star \star \star}$ & $74.6 \pm 3.0^{\star \star \star}$ \\
\hline Caffeine & $89.5 \pm 0.6$ & $90.1 \pm 2.5$ \\
\hline
\end{tabular}

Density analysis of synaptophysin and MAP-2 2 weeks after mutant ataxin-3 transduction. A significant loss of both markers was observed in the water-drinking control group $\left({ }^{* * *} p<0.001\right.$, one-way ANOVA followed by Dunnett's post-hoc test). No loss of either marker was observed in the caffeine-treated group.

Note. Data are expressed as indexes of immunoreactivity of the affected striatal regions relative to their corresponding peripheral non-affected striatum (100\% IR) [mean \pm SEM]. 


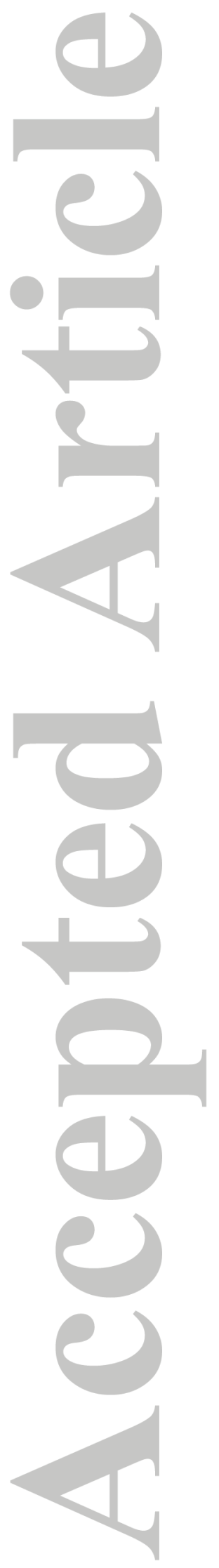

John Wifey \& Sons 


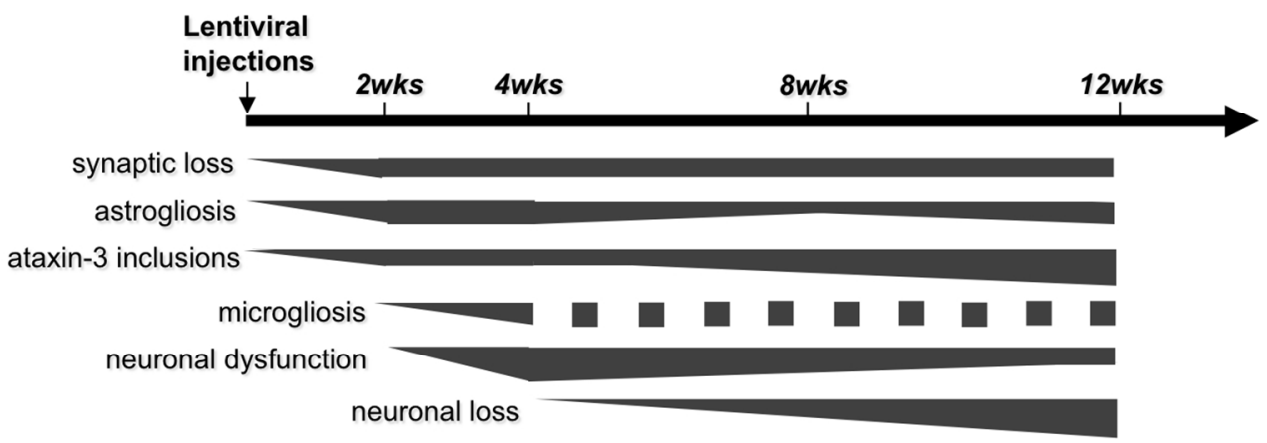

Figure 1: Summary of the time-course of appearance of different morphological features in a lentiviral-based model of Machado-Joseph disease. Loss of synaptic markers and astrogliosis were amongst the most precocious morphological alteration, closely followed by microgliosis and neuronal dysfunction as well as increases of the number of ataxin-3 inclusions, whereas overt neuronal damage occurred later in the development of the disease. Microgliosis would probably be found at later time-points, however, that was not evaluated.

$493 \times 183 \mathrm{~mm}(72 \times 72 \mathrm{DPI})$

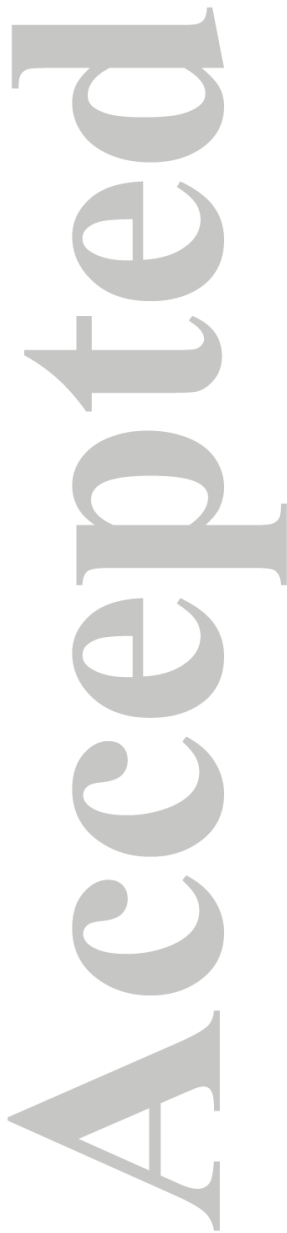



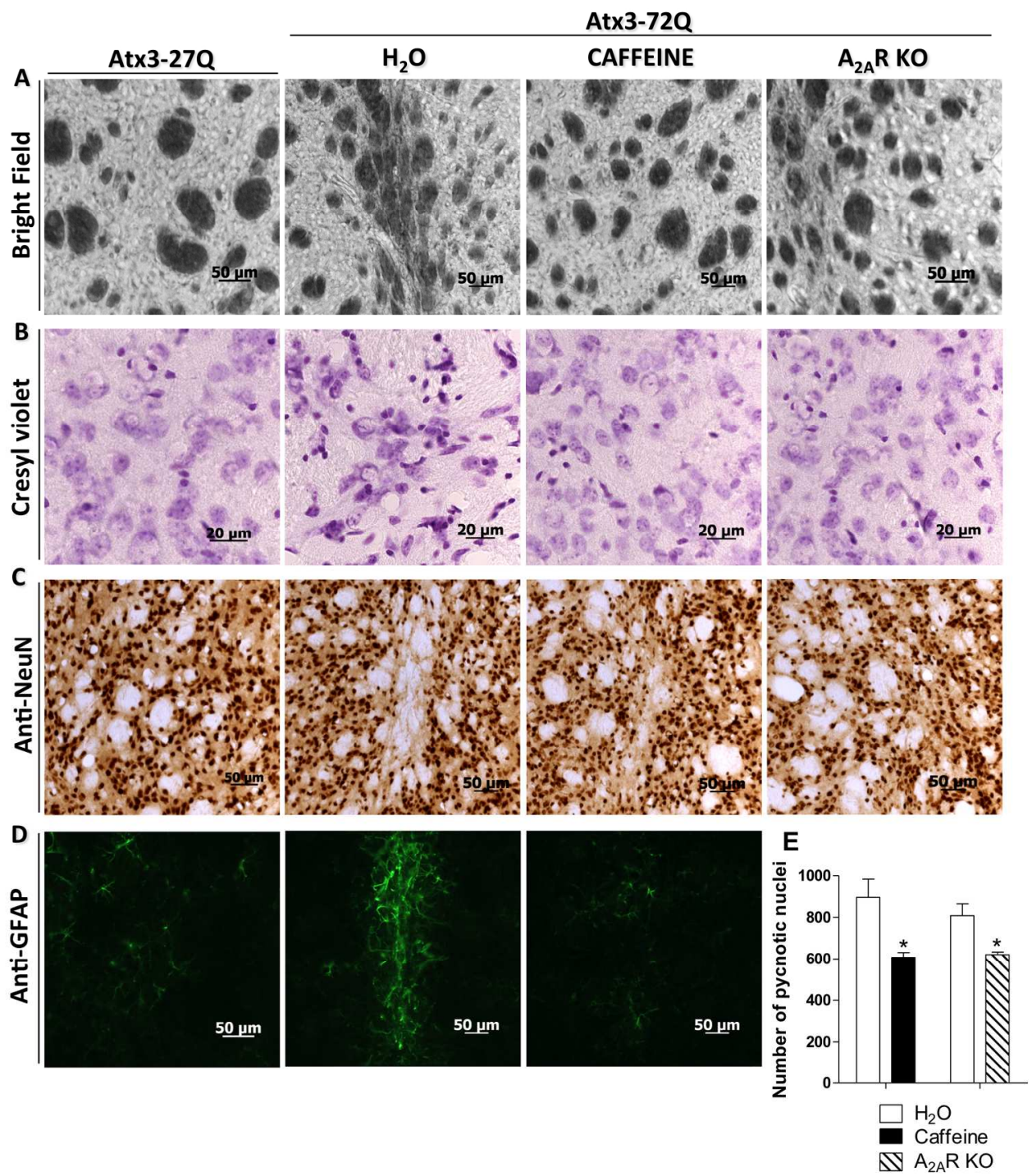

Figure 2: Caffeine treatment or A2AR genetic depletion decreased cell injury and striatal degeneration. Representative bright-field photomicrographs and immunohistochemical stainings from around the injection site area at 12 weeks post-injection of the viral vectors encoding wild-type or mutant ataxin-3. (A) Coalescence of the internal capsule of the striatum was neither seen in the caffeine-treated group nor in the A2AR KO animals. (B) Cresyl violet-stained sections showed a significant reduction of the number of striatal condensated nuclei upon mutant ataxin-3 transduction in the caffeine-treated group as well as in the A2AR $\mathrm{KO}$ group relative to their respective water-drinking groups, as quantified in panel $\mathrm{E}$. (C) A nearly absent loss of neuronal nuclei (NeuN) staining immunoreactivity was seen in the caffeine-treated group as well as in the A2AR KO group. (D) GFAP immunoreactivity showed an increased accumulation of astrocytes (green) replacing neurons after injection of mutant ataxin-3, which was prevented by caffeine consumption. No morphological modifications were detected upon LV-atx3-27Q injections. Statistical significance was evaluated with Student's t test $\left({ }^{*} p<0.05\right)$ comparing both caffeine-drinking and A2AR KO groups with their respective water-drinking wild-type groups upon LV-atx3-72Q injections.

$719 \times 793 \mathrm{~mm}(72 \times 72 \mathrm{DPI})$ 

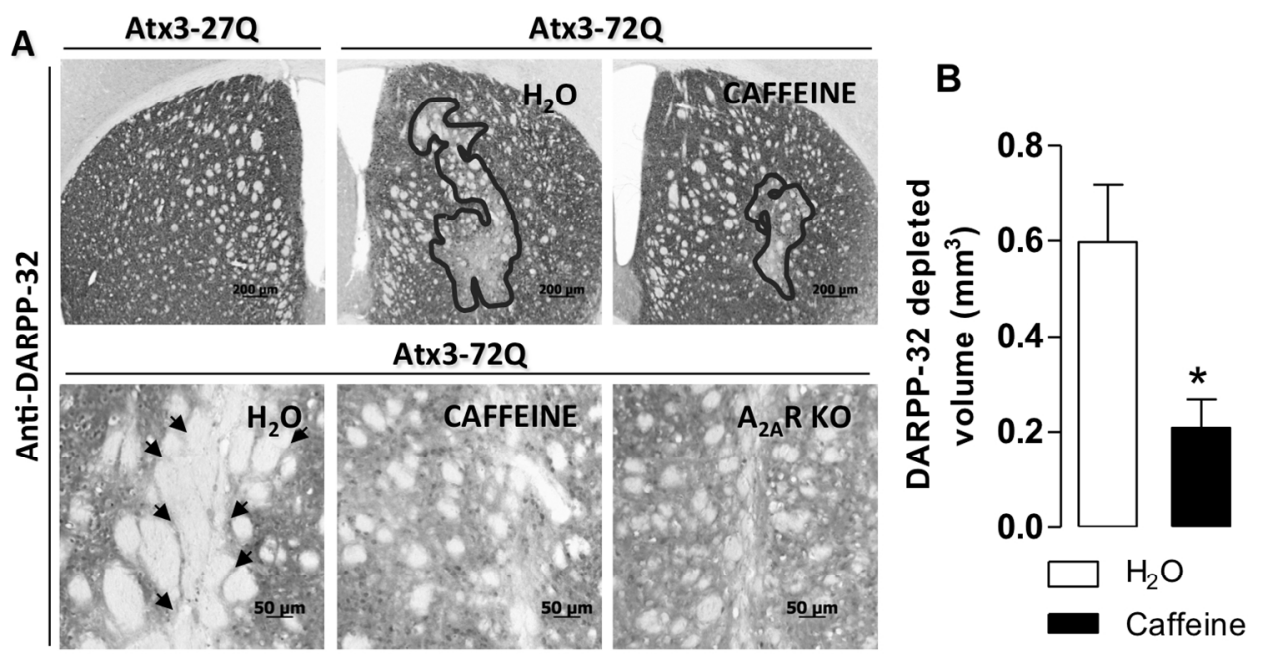

Figure 3: Caffeine treatment or A2AR genetic depletion reduced neuronal dysfunction. ( $A$, upper panel) A large DARPP-32 depleted volume was observed 4 weeks after injection of the viral vectors encoding mutant ataxin-3 in the water-drinking group whereas caffeine-drinking mice exhibited a much smaller lesion area at this time-point. This is quantified in panel $B$ as depleted volume of DARPP-32 staining. No loss of DARPP-32 staining was observed upon LV-atx3-27Q injections. (A, lower panel) At higher magnification, 12 weeks exposure to mutated ataxin-3 revealed a clear condensation of the internal capsule, which joined together fiber patches (arrows). This fiber accumulation was not observed in the caffeine-treated animals or in A2AR KO mice. Statistical significance was evaluated with Student's test $(* p<0.05)$ comparing caffeine-treated $(n=6)$ with water-drinking animals $(n=5)$.

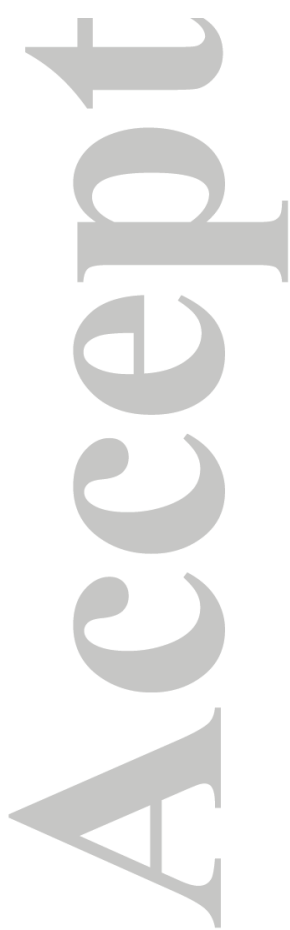
$564 \times 307 \mathrm{~mm}(72 \times 72 \mathrm{DPI})$ 

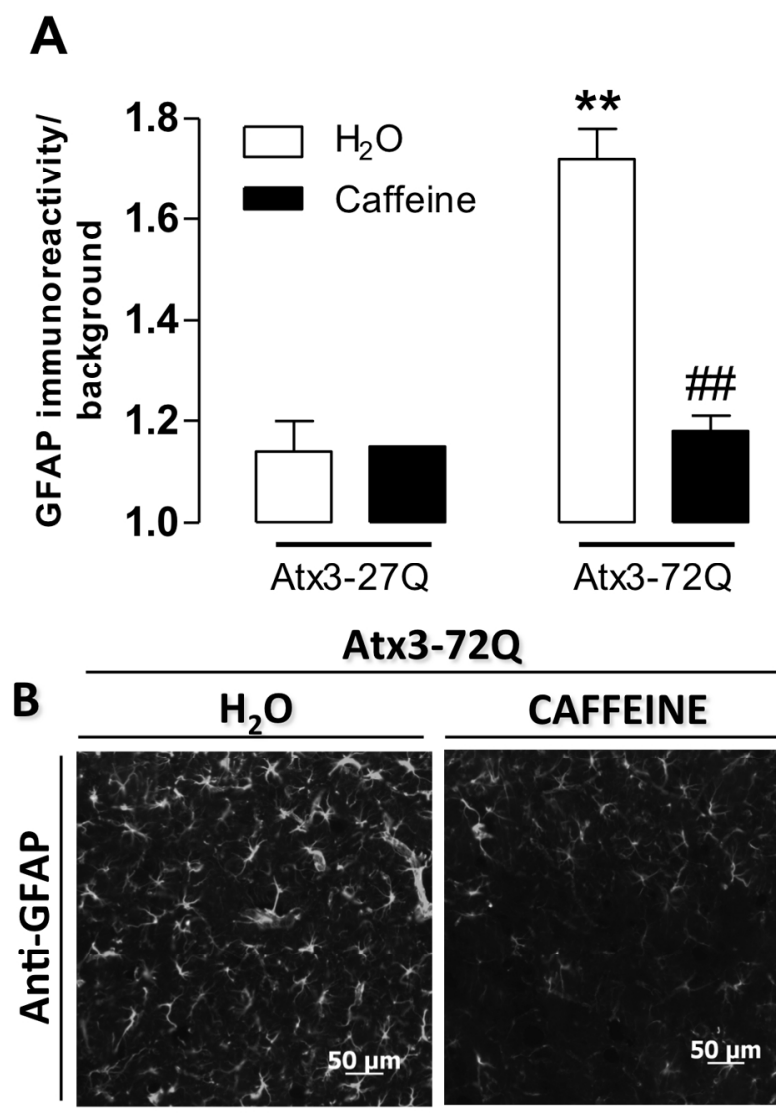

CAFFEINE
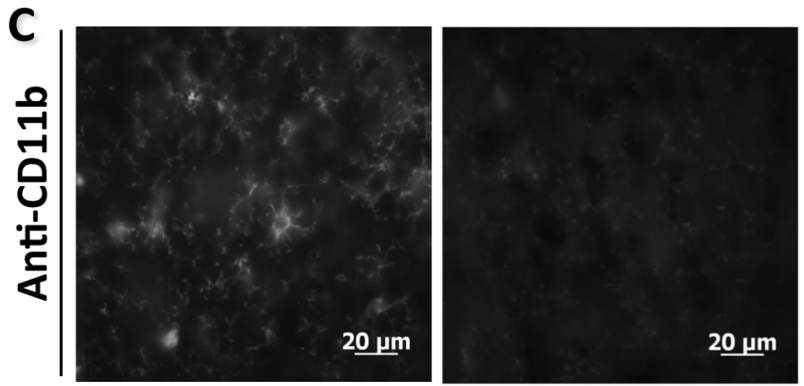

Figure 4: Caffeine treatment decreased MJD-associated astroglial activation and prevented microglia recruitment. (A) Quantification analysis of GFAP immunoreactivity at 4 weeks post-injection of the viral vectors encoding wild-type or mutant ataxin-3. Caffeine abrogated astrocytic activation $(\# \#<0.01)$ to the levels induced by wild-type ataxin-3 (internal control). (B) Caffeine consumption attenuated the mutant ataxin-3-induced activation of astrocytes, gauged by enhanced immunoreactivity of Glial Fibrillary Acidic Protein (GFAP, green) at an earlier time point: 2 weeks. (C) No immunoreactivity for activated microglia, CD11b (Ab 5C6, green), was observed 4 weeks after insult in the caffeine-treated group whereas it was present in water-drinking mice. Statistical significance was evaluated with Student's t test $(* * p<0.01)$ comparing mutant ataxin-3 striatal hemisphere with the contralateral wild-type ataxin-3 hemisphere, and caffeine-drinking with water-drinking groups.

$$
437 \times 793 \mathrm{~mm}(72 \times 72 \mathrm{DPI})
$$



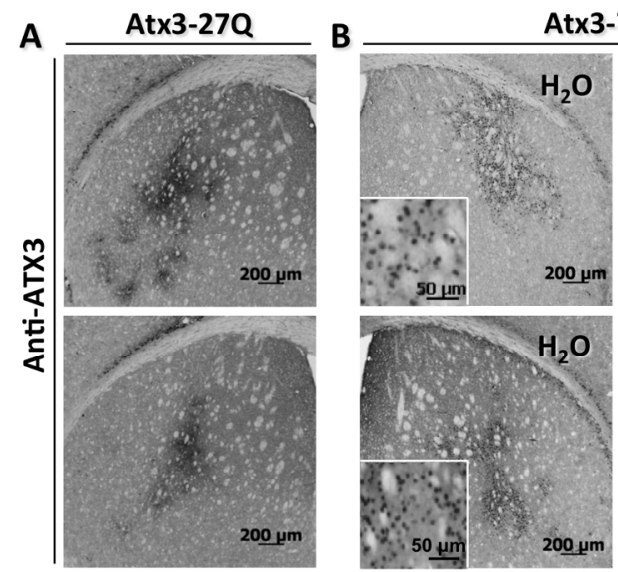

Atx3-72Q
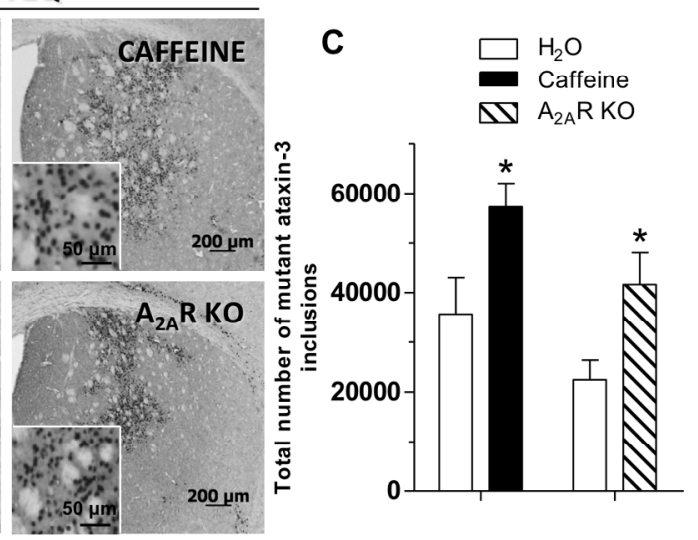

Figure 5: Caffeine treatment or A2AR genetic depletion significantly increased the total number of mutant ataxin-3 inclusions. (A) Using anti-ataxin-3 antibody (Ab 1H9), no nuclear inclusions of ataxin-3 were found on both wild-type and A2AR KO animals upon expression of wild-type ataxin-3. (B) A significant increase in the number of mutant ataxin-3 inclusions was observed 8 weeks post-injection of lentiviral vectors encoding mutant ataxin-3 either in caffeine-treated $(n=5)$ or A2AR KO $(n=5)$ mice compared to the respective waterdrinking wild-type animals transduced with mutant ataxin-3 $(B, n=7 ; E, n=4)$, as quantified in panel $C$. Statistical significance was evaluated with Student's t test $(* p<0.05)$ comparing caffeine-treated and A2AR inactivated animals with their respective water-drinking control groups.

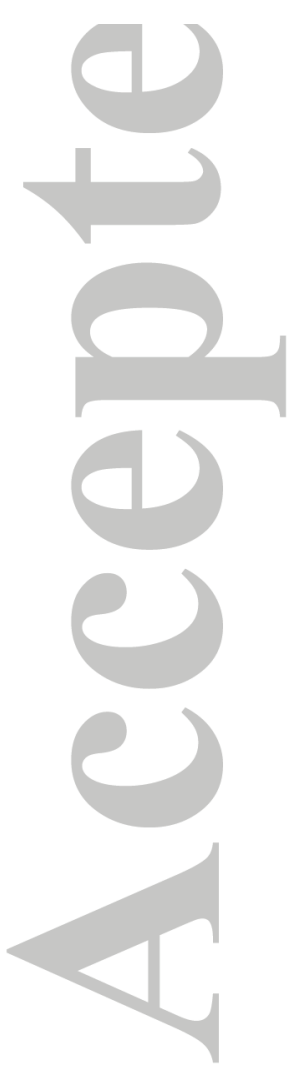
$705 \times 331 \mathrm{~mm}(72 \times 72 \mathrm{DPI})$ 
Atx3-72Q

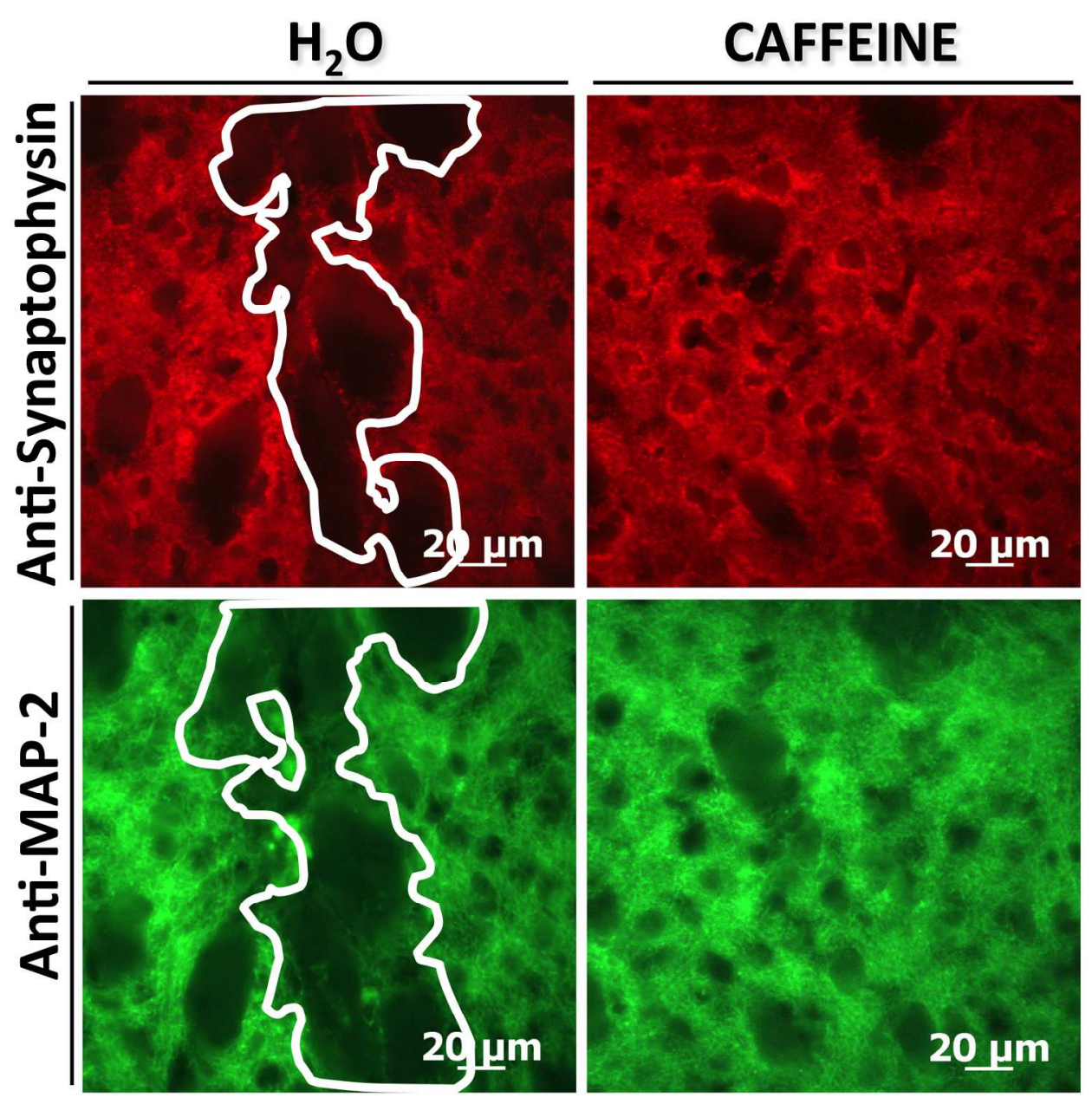

Figure 6: Synaptotoxicity precedes the neurodegeneration process of MJD. Detection of nerve terminals with synaptic markers: synaptophysin (red) and microtubule-associated protein 2 - MAP-2 (Ab AP20, green). No loss of synapses is observed in the group that was given caffeine whereas loss of synaptophysin and MAP-2 immunoreactivity was observed in the non-treated group 2 weeks post-injection of the lentiviral vectors encoding mutant ataxin-3. $737 \times 793 \mathrm{~mm}(72 \times 72 \mathrm{DPI})$ 